\title{
CONTROL OF PASSAGE THROUGH RESONANCE ZONE FOR 1-ROTOR VIBRATION UNIT WITH TIME-VARYING LOAD
}

\author{
O.P. Tomchina \\ Saint Petersburg State University \\ of Architecture and Civil Engineering \\ 4 Vtoraya Krasnoarmeiskaya, 190005 \\ Saint Petersburg, Russia \\ otomchina@mail.ru
}

\author{
D.V. Gorlatov \\ Saint Petersburg State University \\ of Architecture and Civil Engineering \\ 4 Vtoraya Krasnoarmeiskaya, 190005 \\ Saint Petersburg, Russia \\ dgorlatov@lan.spbgasu.ru
}

\author{
D.A. Tomchin \\ Institute of Problems in Mechanical Engineering \\ 61 Bolshoy ave. V.O., Saint Petersburg, 199178, Russia \\ tda@ipme.ru
}

\author{
A.E. Epishkin \\ Saint Petersburg State University \\ of Architecture and Civil Engineering \\ 4 Vtoraya Krasnoarmeiskaya, 190005 \\ Saint Petersburg, Russia \\ epishkin@mail.ru
}

Article history:

Received 25.08.2021, Accepted 27.09.2021

\begin{abstract}
The speed-gradient algorithms for controlled passage through the resonance zone of the one-rotor vibration unit are studied by computer simulation. The objective of the study is to analyze dependence of the control performance on the loading mode and the electric drive dynamics. In order to obtain an algorithm better suitable for practical implementation the theoretically designed algorithm is simplified by simplifying the expression for the total energy. First of all, we neglect the terms corresponding to the kinetic and potential energy of the load, since there are no load mass sensors on the stand. the term containing the inclination angle of the platform is neglected. In addition, the platform inclination angle and dynamics of the drives were neglected too. Efficiency of the proposed simplified algorithm for different loading modes, including linear loading with different loading rates and sine-shaped oscillatory loading.
\end{abstract}

\section{Introduction}

An important role in mechanics is played by the methods of vibration mechanics pioneered and developed by Iliya Izrailevich Blekhman and his successors [Blekhman, 2000]. I.I.Blekhman suggested to extend those methods to a broader area of dynamical systems of any origin [Blekhman, 2012]. Based on these achievements I.I.Blekhman and his colleagues have formulated main problems of control for vibration machines: control of start-up, control of synchronization, control of passage through resonance zone. First solutions to those problems were summarized in the book [Andrievsky et al., 2001].

A possibility of control of passage through resonance zone is an interesting phenomenon in nonlinear mechanics related to overcoming of the so called Sommerfeld effect. In [Tomchina, 1997] a new approach to control of passage through resonance zone for 1-rotor vibration unit, based on the speed-gradient method [Fradkov, 1980], [Fradkov et al., 2021] was proposed. It was extended to two-rotor vibration machines [Fradkov et al., 2014]. Later flexibility of the motor shafts [Tomchin et al., 2015] and dynamics of the drives [Gorlatov et al., 2015] were taken into account.

In this paper the method proposed in [Tomchina, 1997] is studied for time-varying modes of the units arising when the load of the unit is time-varying.

\section{Model of one-rotor vibration unit taking into ac- count the drive dynamics}

To study the efficiency of control algorithms for the main modes of vibration units the mechatronic vibration setups with one unbalanced rotor were developed [Blekhman et al., 1999]. Dynamics of one-rotor vibration units without taking payload and electric drives into account were studied in [Fradkov et al., 2016]. The 


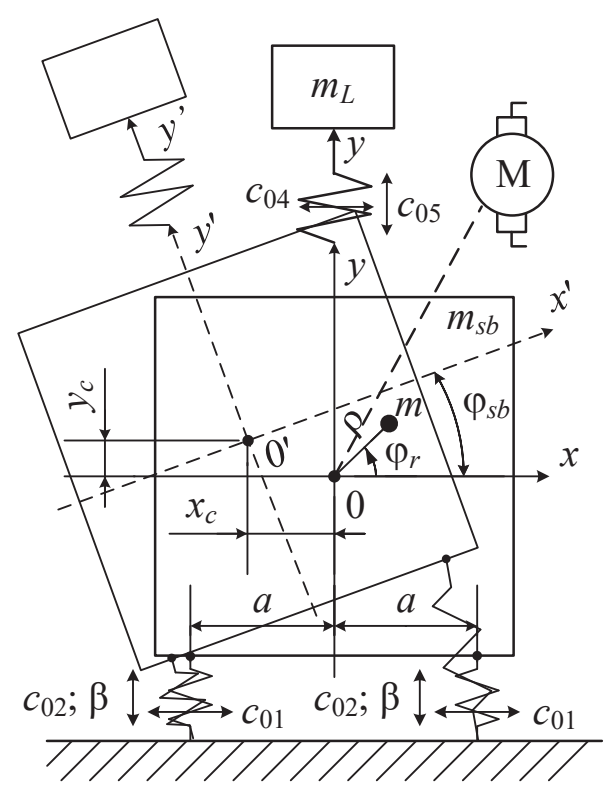

Figure 1. Schematics of one-rotor vibration unit with DC motor and the load.

schematics of one-rotor vibration unit with DC motor and the load, whose supporting body is oscillating in a vertical plane $0 x y$ is shown in Fig. 1. Here, a coordinate system associated with the supporting body is designated as $0^{\prime} x^{\prime} y^{\prime}$. The coordinates of the center of the supporting body - point $0^{\prime}$ in the fixed coordinate system are denoted as $\left\{x_{c}, y_{c}\right\}$. The rotor is located in the center of the supporting body. The supporting body is reinforced by elastic supports - springs located at $x_{n i}= \pm a$. The rotation angle of the supporting body is denoted as $\varphi_{s b}$. In addition, the following notation is used: $\varphi_{r}$ - rotation angle of an unbalanced rotor, measured from the axis $0 x^{\prime}$ counterclockwise; $m_{s b}, m, m_{L}$ - the masses of the supporting body, the rotor and the load ; $J$ - moment of inertia of the rotor; $\varrho$ - eccentricity of the rotor; $c_{01}, c_{04}$ - longitudinal rigidities and $c_{02}, c_{05}$ lateral rigidities of the springs; $c_{03}=-2 \alpha^{2}\left(c_{02}-c_{01}\right) ; g-$ acceleration of gravity; $k_{c}$ - viscous friction coefficient of the vibroactuator bearings; $\beta-$ axial damping of springs. It is assumed that rotor shaft is orthogonal to the motion of the support. Let $M_{m}$ be control action (torque of the drive motor), $m_{0}=m_{s b}+m$. Total, potential and kinetic mechanical energies of the system respectively are as follows:

$$
\begin{aligned}
& H=T+\Pi ; \\
& T=0.5 m_{0} \dot{x}_{c}^{2}+0.5 m_{0} \dot{y}_{c}^{2}+0.5 J \dot{\varphi}_{r}^{2}+0.5 m \varrho \dot{\varphi}_{s b} \dot{\varphi}_{r}- \\
& x_{c} \dot{\varphi}_{s b} m \varrho \sin \left(\varphi_{s b}+\varphi_{r}\right)-x_{c} \dot{\varphi}_{r} m \varrho \sin \left(\varphi_{s b}+\varphi_{r}\right)+ \\
& y_{c} \dot{\varphi}_{s b} m \varrho \cos \left(\varphi_{s b}+\varphi_{r}\right)+y_{c} \dot{\varphi}_{r} m \varrho \cos \left(\varphi_{s b}+\varphi_{r}\right)+ \\
& 0.5 m_{L}\left(\dot{x}_{L}^{2}+\dot{y}_{L}^{2}\right) ; \\
& \Pi=m_{0} g y_{c}+m g \varrho \sin \left(\varphi_{s b}+\varphi_{r}\right)+ \\
& c_{01}\left(x_{c}^{2}+\alpha^{2} \cos ^{2} \varphi_{s b}\right)+c_{01}\left(y_{c}^{2}+\alpha^{2} \sin ^{2} \varphi_{s b}\right)+ \\
& m_{L} g y_{L}+0.5 c_{04}\left(x_{c}-x_{L}\right)^{2}+m_{L} g y_{L}+ \\
& 0.5 c_{05}\left(y_{c}-y_{L}\right)^{2} .
\end{aligned}
$$

Since the work studies the comparative efficiency of control using a special algorithm for passing through resonance for various models of one-rotor vibration unit, shown in Fig. 1, then this section presents the equations of dynamics for all these models.

The equations of dynamics for model M1 without taking into account the influence of the load and electric drives have the following form:

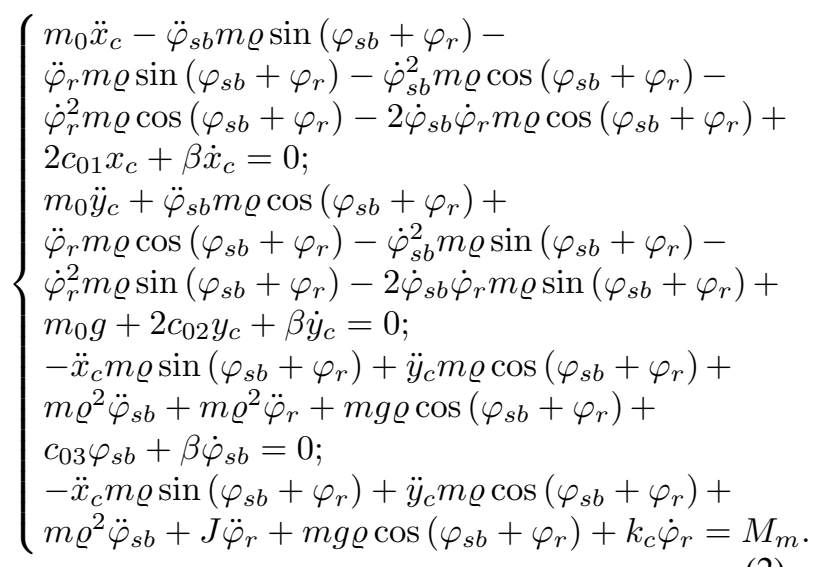

Since the laboratory setup SV employs the DC motor, the electric drive structure is selected as the traditional single-circuit system with current loop and proportionalintegral (PI) current controller $W_{C R}(p)=b(\tau p+1) / \tau p$ is configured to optimum modulo; $b, \tau$ are dynamic gain and time constant of the regulator. The following notation is used here: CR is the current regulator; TC is the power (thyristor type) converter; CS is the current sensor; $I_{a}$ is the armature current; $E_{T C}$ and $E_{m}$ are converter and motor EMFs; $k_{T C}$ and $k_{C S}$ are converter and current feedback gains; $k F$ is the motor torque (EMF) coefficient; $T_{T C}$ and $T_{C S}$ are converter and current sensor time constants; $T_{a}$ is the armature time constant; $R_{a}$ is the armature circuit resistance; $U_{C R}$ and $U_{C S}$ are current controller and current sensor output voltages.

Motor torque $M_{m}$ is the solution of the system of differential and algebraic equations

$$
\begin{aligned}
\dot{I}_{a} & =\frac{1}{T_{a}}\left(-I_{a}+\frac{1}{R_{a}}\left(E_{T C}-E_{m}\right)\right) \\
\dot{E}_{T C} & =\frac{1}{T_{T C}}\left(-E_{T C}+k_{T C} U_{C R}\right) \\
\dot{U}_{C S} & =\frac{1}{T_{C S}}\left(-U_{C S}+k_{C S} I_{a}\right) \\
\dot{U}_{C S 1} & =\frac{b}{\tau}\left(U-U_{C S}\right) \\
U_{C S} & =b\left(U-U_{C S}\right)+U_{C S 1}, \quad \\
E_{m} & =k F \dot{\varphi}, \quad M_{m}=k_{m} I_{a}, \quad U=k_{U} M
\end{aligned}
$$

where $U$ is the voltage corresponding to the calculated torque $M$, obtained in accordance with the equations (6), $k_{m}=k F$. Thus, the dynamics model of one-rotor vibration unit with DC motor M2 is described by differential equations (2) - (3). 
The equations of dynamics for the model taking into account the load and electric drives M3 are described by the differential equations (3), (4).

$$
\left\{\begin{array}{l}
m_{0} \ddot{x}_{c}-\ddot{\varphi}_{s b} m \varrho \sin \left(\varphi_{s b}+\varphi_{r}\right)- \\
\ddot{\varphi}_{r} m \varrho \sin \left(\varphi_{s b}+\varphi_{r}\right)-\dot{\varphi}_{s b}^{2} m \varrho \cos \left(\varphi_{s b}+\varphi_{r}\right)- \\
\dot{\varphi}_{r}^{2} m \varrho \cos \left(\varphi_{s b}+\varphi_{r}\right)-2 \dot{\varphi}_{s b} \dot{\varphi}_{r} m \varrho \cos \left(\varphi_{s b}+\varphi_{r}\right)+ \\
2 c_{01} x_{c}+\beta \dot{x}_{c}+c_{04}\left(x_{c}-x_{l}\right)=0 \\
m_{0} \ddot{y}_{c}+\ddot{\varphi}_{s b} m \cos \left(\varphi_{s b}+\varphi_{r}\right)+ \\
\ddot{\varphi}_{r} m \varrho \cos \left(\varphi_{s b}+\varphi_{r}\right)-\dot{\varphi}_{s b}^{2} m \varrho \sin \left(\varphi_{s b}+\varphi_{r}\right)- \\
\dot{\varphi}_{r}^{2} m \varrho \sin \left(\varphi_{s b}+\varphi_{r}\right)+m_{0} g- \\
2 \dot{\varphi}_{s b} \dot{\varphi}_{r} m \varrho \sin \left(\varphi_{s b}+\varphi_{r}\right)+2 c_{02} y_{c}+\beta \dot{y}_{c}+ \\
c_{05}\left(y_{c}-y_{L}\right)=0 \\
-\ddot{x}_{c} m \varrho \sin \left(\varphi_{s b}+\varphi_{r}\right)+\ddot{y}_{c} m \varrho \cos \left(\varphi_{s b}+\varphi_{r}\right)+ \\
m \varrho^{2} \ddot{\varphi}_{s b}+m^{2} \ddot{\varphi}_{r}+m g \varrho \cos \left(\varphi_{s b}+\varphi_{r}\right)+ \\
c_{03} \varphi_{s b}+\beta \dot{\varphi}_{s b}=0 ; \\
-\ddot{x}_{c} m_{\sin }\left(\varphi_{s b}+\varphi_{r}\right)+\ddot{y}_{c} m \varrho \cos \left(\varphi_{s b}+\varphi_{r}\right)+ \\
m \varrho^{2} \ddot{\varphi}_{s b}+J \ddot{\varphi}_{r}+m g \varrho \cos \left(\varphi_{s b}+\varphi_{r}\right)+k_{c} \dot{\varphi}_{r}= \\
=k_{m} I_{a} \\
m_{L} \ddot{x}_{L}-c_{04}\left(x_{c}-x_{L}\right)+\dot{m}_{L} \dot{x}_{L}+\beta \dot{x}_{L}=F_{x}, \\
m_{L} \ddot{y}_{L}+\dot{m}_{L} \dot{y}_{L}+m_{L} g-c_{05}\left(y_{c}-y_{L}\right)+\beta \dot{y}_{L}=F_{y},
\end{array}\right.
$$

where the coordinates of the center of the load are denoted as $\left\{x_{L}, y_{L}\right\}, F_{x}, F_{y}$ are projections on the $0 x$ and $0 y$ axes, respectively, of the resultant external forces $F$ acting on the load, and due to the speed and mass of the screened material falling from the unloading end of the unit.

\section{The algorithm for controlled passage through res- onance zone}

The idea of of speed-gradient approach to controlled passage through resonance zone [Tomchina, 1997] is to extract the slow motion $\Psi(t)$ and to swing it with the aim to increase the energy of the rotating subsystem. To extract slow motions, low-pass filter is inserted into the energy control algorithms. Particularly, if slow component appears in oscillations of rotor angular velocity $\dot{\varphi}_{r}$, then the control algorithm proposed in [Tomchina, 1997] is used:

$$
M=-\gamma \operatorname{sign}\left(\left(H-H^{*}\right) \dot{\psi}\right), T_{\psi} \dot{\psi}=-\psi+\dot{\varphi}_{r},
$$

where $H$ denotes total mechanical energy of the system; $\psi(t)$ is the variable of a filter, that is an estimate of the slow motion $\Psi(t) ; T_{\psi}$ is the time constant of a filter, $\gamma>0$ is constant. At low damping, slow motions decay slowly, and it gives to the control algorithm an opportunity to create suitable conditions to pass through resonance zone. Thus the effect of "feedback resonance" [Fradkov, 1999] is created. After passing the resonance zone it is suggested to turn off the "swinging" and then to switch the algorithm to controlling with constant torques.

In [Gorlatov et al., 2015] it was proposed to include a switch (time varying gain $\gamma(t)$ ) into the algorithm (5) providing cancellation of control signal after passage of the rotor angular velocity through resonance zone. The algorithm with a switch is implemented as follows:

$$
\left\{\begin{array}{l}
M=\left\{\begin{array}{l}
M_{0}, \text { if } \gamma_{1}(t)=1, \\
M_{0}, \text { if } \gamma_{1}(t)=0 \&\left(H-H^{*}\right)\left(\dot{\varphi}_{r}-\psi\right)>0, \\
0, \text { else }
\end{array}\right. \\
T_{\psi} \dot{\psi}=-\psi+\dot{\varphi}_{r}
\end{array}\right.
$$

where $H$ is total mechanical energy of the system; $\psi(t)$ is the variable of the filter; $T_{\psi}>0, T_{\psi}$ and $H^{*}$ are the parameters of the algorithm; $\gamma_{1}(t)=\max _{0 \leq \tau<t} \operatorname{sgn}(H(\tau)-$ $\left.H^{*}\right)$, where $\operatorname{sgn}[z]=1$ with $z>0 ; \operatorname{sgn}[z]=0$ with $z \leq 0$.

Since the expression for the total mechanical energy $H$ has a rather cumbersome form, it is worth to reduce it to simplify the calculation of the control torque. The legitimacy of this simplification will be investigated using computer simulations. The need for such a simplification is dictated by the available sensors and other possibilities for recovering coordinates, in particular, the coordinates and parameters of the load. The greatest difficulty is the measurement or assessment with the help of observers of the angle of rotation. In addition, in steadystate modes, the value is insignificant. Therefore, it is possible to simplify the expression for the total energy by setting $\varphi_{s b}=0$. In addition, we neglect the terms corresponding to the kinetic and potential energy of the load, since there are no load mass sensors on the stand. We also consider that the horizontal movement sensors of the platform are not involved. Then the expression for mechanical energy in the algorithm is as follows:

$$
\left\{\begin{array}{l}
\tilde{H}=\tilde{T}+\tilde{\Pi} \\
\tilde{T}=0.5 m_{0} \dot{y}_{c}^{2}+0.5 J \varphi_{r}^{2}+\dot{y}_{c} \dot{\varphi}_{r} m \varrho \cos \varphi_{r} \\
\tilde{\Pi}=m_{0} g y_{c}+m g \varrho \sin \varphi_{r}+c_{02} y_{c}^{2},
\end{array}\right.
$$

and the modified algorithm for passing through resonance using expression (7) takes the form

$$
\left\{\begin{array}{l}
M=\left\{\begin{array}{l}
M_{0}, \text { if } \gamma_{1}(t)=1, \\
M_{0}, \text { if } \gamma_{1}(t)=0 \&\left(\tilde{H}-H^{*}\right)\left(\dot{\varphi}_{r}-\psi\right)>0, \\
0, \text { else }
\end{array}\right. \\
T_{\psi} \dot{\psi}=-\psi+\dot{\varphi}_{r},
\end{array}\right.
$$

The filter time constant $T_{\psi}$ must exceed the period of the resonant oscillations. At the same time, too large values lead to a decrease in the average power of the control signal and inhibition of the algorithm. The final choice of the characteristics of the $T_{\psi}, \gamma$ algorithm is carried out according to the results of computer simulation.

\section{Computer simulation results}

The simulation was carried out in three stages:

1. Investigation of the algorithm for the passage through the resonance zone for the model without taking into account the dynamics of the electric drive and the load (M1); 


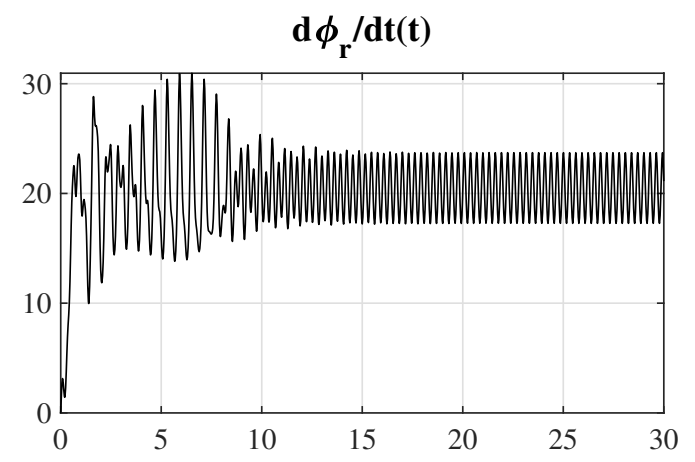

Figure 3. Results of modeling a model with an electric drive at direct start for $M_{m}=1.7 \mathrm{Nm}$.

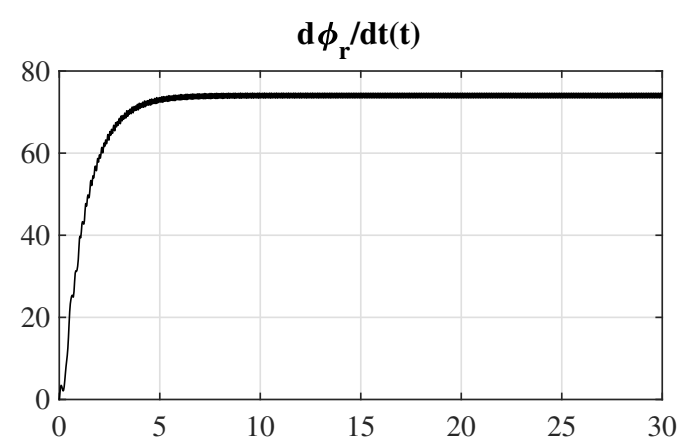

Figure 2. Simulation results of the model (M2) with direct start for $M_{m}=1.8 \mathrm{Nm} ; \dot{\varphi}_{r m}(\infty)=75 \mathrm{~s}^{-1}$.

2. Investigation of the algorithm for the passage through the resonance zone for the model, taking into account the dynamics of the electric drive (M2);

3. Investigation of the algorithm for the passage through the resonance zone for the model, taking into account the dynamics of the electric drive and the dynamics of an elastically attached load (M3).

First of all, the value of the control torque was determined, which ensures the passage through the resonance during direct start using a constant electromechanical torque (i.e., without using a special algorithm (8)). Since the main model studied in this article is the model with electric drive (M2), the graphs of the rotor speed for this particular model are presented. Figure 2 shows the simulation results for direct start-up for a torque of $M_{m}=1.8 \mathrm{Nm}$. As can be seen from the graph, the rotor speed goes into the over-resonance region and fluctuates around the level of $75 \mathrm{~s}^{-1}$. Note that with direct start, the operating speed of the rotor cannot be made less than this value.

Figure 3 shows the simulation results for the model (M2) with direct start for $M_{m}=1.7 \mathrm{Nm}$. As can be seen from the graph, the rotor speed does not go into the over-resonant region and fluctuates near the resonance frequency of $22 \mathrm{~s}^{-1}$. Thus, for this model, the minimum torque at which the rotor speed passes resonance during direct start is $M_{m}=1.8 \mathrm{Nm}$.
At the first stage, the control efficiency was investigated using the algorithm for passing the resonant frequencies (8) for the model without taking into account the dynamics of the electric drive and the load (M1). Note that for this model, the minimum torque at which the rotor speed passes resonance during direct start is $M_{m}=0.68 \mathrm{Nm}$, and the corresponding speed value $\dot{\varphi}_{r m}(\infty)=70 \mathrm{~s}^{-1}$. Obviously, the value of this torque for the model (M1) is less than in the second case.

As shown by the study of the proposed algorithm for passing through the resonance for the model (M1), the average speed of the rotor $\dot{\varphi}_{r m}(\infty)$ after passing the resonant frequency is stabilized at a certain constant level $\omega_{r}^{*}$, corresponding to the energy $H^{*}$ specified in the algorithm. In this case, this speed $\dot{\varphi}_{r m}(\infty)$ is determined only by the parameter of the algorithm $H^{*}$, and the coefficients $\gamma$ and $T_{\psi}$ affect only the transient time of the resonance $t_{\text {pas }}$ and transient time for the rotor speed $t_{t r}$. Figure 4 shows the simulation results corresponding to the first stage of the study, when controlled using algorithm (8).
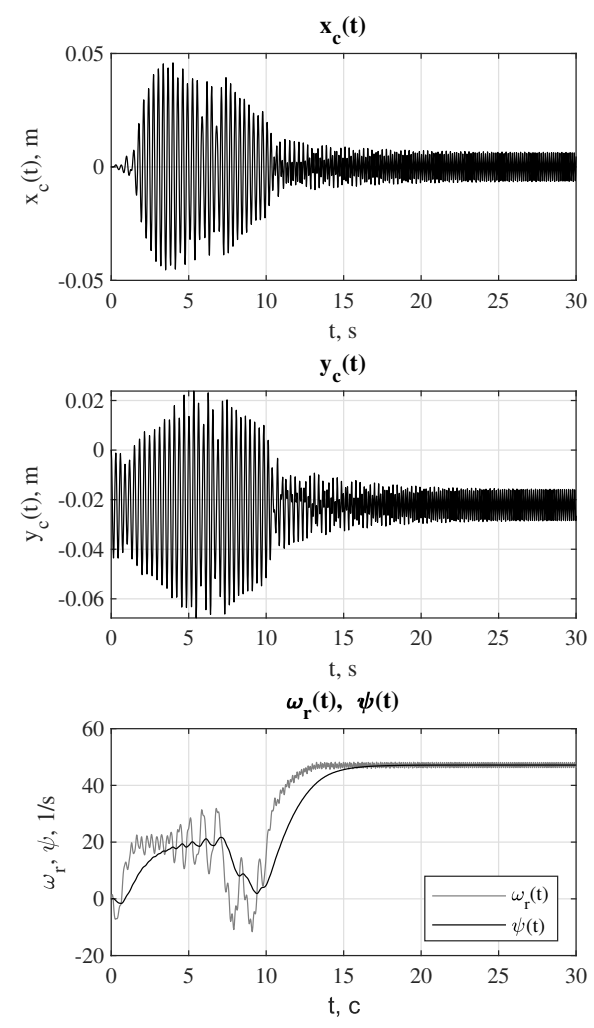

Figure 4. Simulation results for the model (M1) using the algorithm (8) at $M_{m}=0.5 \mathrm{Nm}, H^{*}=14 \mathrm{~J}$.

Fig. 4 corresponds to the model (M1) with the following nominal system parameters: $J_{1}=J_{2}=$ 

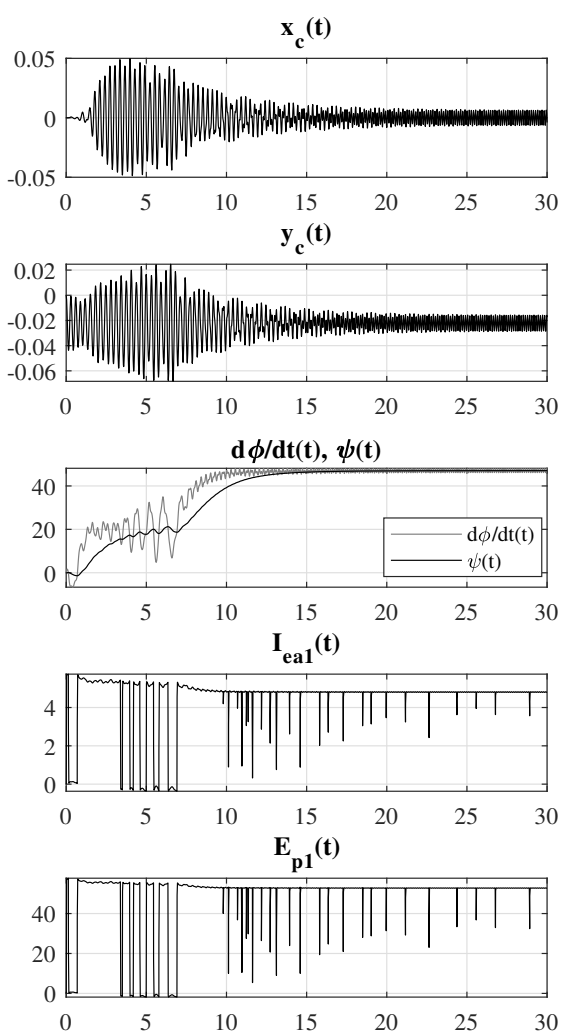

Figure 5. Results of simulation for the model (M2) using algorithm (8) for $M_{m}=1.15 \mathrm{Nm}, H^{*}=14 \mathrm{~J}$.
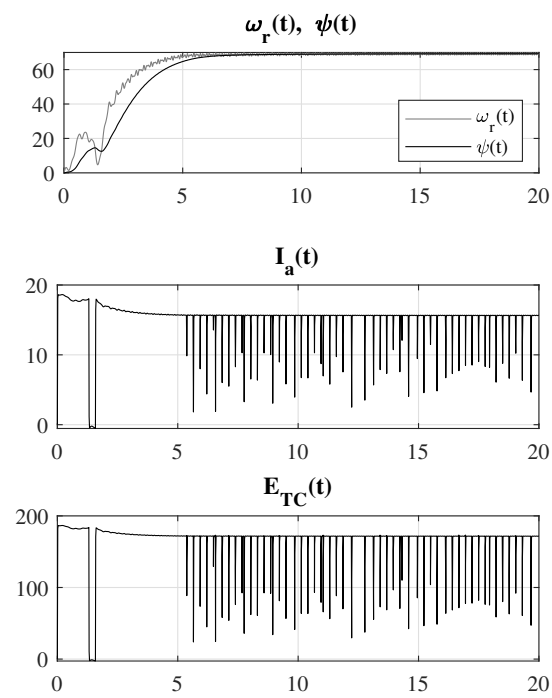

Figure 6. Results of simulation for the model (M2) using algorithm (8) for $M_{m}=1.6 \mathrm{Nm}, H^{*}=65 \mathrm{~J}$.

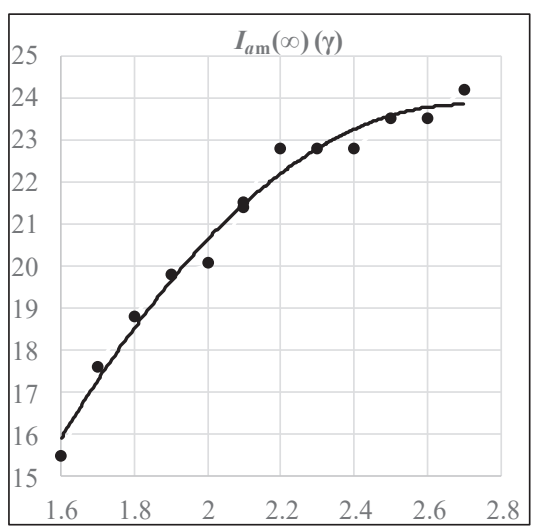

Figure 7. Dependence of the steady-state armature current amplitude $I_{a m}(\infty)$ on parameter of the algorithm $\gamma$ (polynomial approximation).

$0.014 \mathrm{~kg} \cdot \mathrm{m}^{2} ; m=1.5 \mathrm{~kg} ; m_{s b}=9 \mathrm{~kg} ; \varrho=0.04 \mathrm{~m} ;$ $k_{c}=0.01 \mathrm{~J} / \mathrm{s}, \beta=5 \mathrm{~kg} / \mathrm{s}, c_{01}=1300 \mathrm{~N} / \mathrm{m}, c_{02}=5300$ $\mathrm{N} / \mathrm{m}, H^{*}=14 \mathrm{~J}, \gamma=0.5 ; T_{\psi}=0.1 \mathrm{~s}$. The gain $\gamma$ is numerically equal to the value of the control torque $M_{m}$.

The dynamics of the following variables are presented in Fig 4: the horizontal and vertical displacements of the supporting body $x_{c}(t), y_{c}(t)$, rotor velocity $\omega_{r}=\dot{\varphi}_{r}(t)$ and filter coordinate $\psi(t)$.

As is seen from the plot $\dot{\varphi}_{r m}(\infty)=47.5 \mathrm{~s}^{-1}$. Thus, the level of electromechanical torque, which ensures the passage of resonance for model (M1), using algorithm (8) can be reduced by more than $25 \%$.

At the second stage, the algorithm for the passage through resonance zone for the model was investigated, 


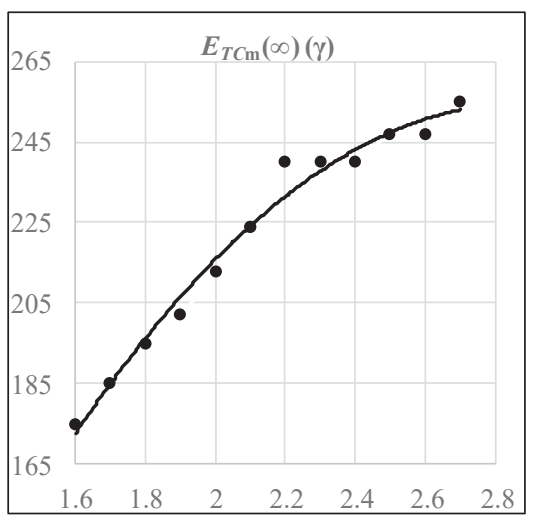

Figure 8. Dependence of the steady-state EMF amplitude of the converter $E_{T C m}(\infty)$ on parameter of the algorithm $\gamma$.

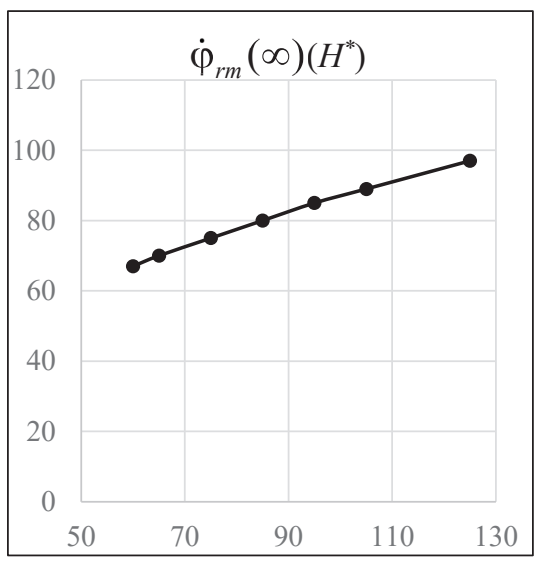

Figure 9. Dependence of the steady-state rotor speed on the value of the given energy $H^{*}$ for model (M2).

taking into account the dynamics of the electric drive (M2). Figure 5 shows the simulation results for the model (M2) using algorithm (8) for the same energy value as in Fig. 4: $H^{*}=14 \mathrm{~J}$. The resonance is overcome here at a larger value of the algorithm gain $\gamma=1.15$, i.e. at $M_{m}=1.15 \mathrm{Nm}\left(T_{\psi}=1.2 \mathrm{~s}\right)$. As can be seen from the graph, the steady-state average rotor speed, as in the first case, $\dot{\varphi}_{r m}(\infty)=47.5 \mathrm{~s}^{-1}$, $t_{\text {pas }}=7.38 \mathrm{~s}, t_{t r}=10.35 \mathrm{~s}$. In addition to the graphs of the variables shown in Fig. 4, Fig. 5 shows graphs of changes in the armature current $I_{a}$ and the EMF of the converter $E_{T C}$.

Figure 6 shows the simulation results for the same model with other parameters of the algorithm: $H^{*}=65$ $\mathrm{J}, \gamma=1.6 ; T_{\psi}=0.1 \mathrm{~s}$.

The quantitative characteristics of the efficiency of the algorithm for passing through the resonance for the second stage are presented in Table 1. The table is obtained for $H^{*}=65 \mathrm{~J}$, which corresponds to the steady-state average rotor speed $\dot{\varphi}_{r m}(\infty)=70 \mathrm{~s}^{-1}$. The table shows $\gamma, T_{\psi}$ - the parameters of the algorithm for passing through the resonance, $t_{\text {pas }}$ - the time of passage of the resonance, and the steady-state values of the amplitude of oscillations of the armature current $I_{a m}(\infty)$ and the EMF of the converter $E_{T C m}(\infty)$.

The data in Table 1 allow us to analyze the influence of the parameter $\gamma$ of the algorithm on the steady-state amplitude of the armature current and the EMF of the converter. The indicated dependences are shown in Fig. 7 and Fig. 8, respectively.

Figure 9 shows the dependence of the steady-state rotor speed on the value of the given energy $H^{*}$ in the range from $60 \mathrm{~J}$ to $125 \mathrm{~J}$.

Thus, as shown by simulation with control using algorithm (8), the level of electromechanical torque, which ensures the passage of resonance for the model (M2), taking into account the dynamics of the drive, can be reduced by more than $30 \%$ compared to direct start.

The constructed dependence of the value of the steadystate speeds of the rotors on the level of the given energy makes it possible to determine the value of the specified parameter in the control algorithm for practical implementation, depending on the technological task. In addition, simulation has shown that the values of steady-state speed do not depend on the parameters $\gamma$ and $T_{\psi}$, but are determined only by the value of the given total energy $H^{*}$.

However, the parameters $\gamma$ and $T_{\psi}$ significantly affect the amplitude of the steady-state armature current and the steady-state EMF of the converter. In this case, an increase in the parameter $\gamma$ leads to an increase in the amplitude of the current and EMF, which is impractical, since this parameter does not affect the steady-state rotor speed. The influence of the values of $\gamma$ and $T_{\psi}$ on the time of passage through the resonance is insignificant, since the specified time varies from $2 \mathrm{~s}$ to $5 \mathrm{~s}$ and acceleration to the set operating speed is carried out in a time not exceeding $10 \mathrm{~s}$.

At the third stage, the efficiency of the algorithm for the passage of resonant frequencies for the model was investigated, taking into account the dynamics of the electric drive and the dynamics of a variable elastic-attached load (M3).

The change in the weight of the load during the simulation was carried out according to the following scheme. At the initial time interval $\left[0 ; t_{1}\right]$ it was assumed that the load is constant, but not equal to zero (this emulates an empty pallet for bulk material, which is installed on the bench). The time $t_{1}$ is longer than the transient process time for the rotor speed, that is, the loading process begins after the vibration unit enters the operating mode. After that, the mass of the load begins to increase loading is in progress. The load is considered as a resiliently attached point mass. At time $t_{2}$, it is assumed that the average amount of bulk material being poured is approximately equal to the amount of material leaving 

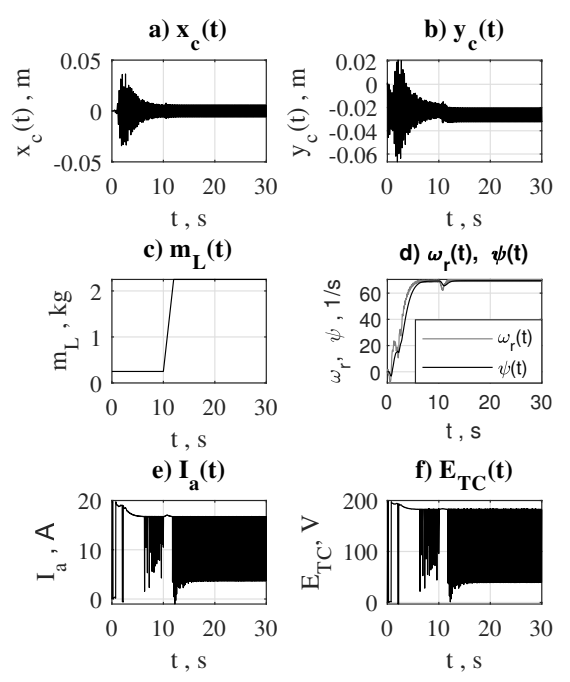

Figure 10. Simulation results for the model with variable load (M3) using algorithm (8) for $V_{0}=1,0 \mathrm{~kg} / \mathrm{s} ; t_{1}=10 \mathrm{~s} ; t_{2}=12 \mathrm{~s}$; $m_{L m}=2,25 \mathrm{~kg}$.

Table 2 .

\begin{tabular}{|c|c|c|c|c|c|}
\hline $\begin{array}{c}V_{0}, \\
\mathrm{~kg} / \mathrm{s}\end{array}$ & $\begin{array}{c}\Delta V, \\
\mathrm{~kg} / \mathrm{s}\end{array}$ & $\begin{array}{c}t_{1}, \\
\mathrm{~s}\end{array}$ & $\begin{array}{c}t_{2}, \\
\mathrm{~s}\end{array}$ & $\begin{array}{c}m_{L m}, \\
\mathrm{~kg}\end{array}$ & $\begin{array}{c}\Delta \omega_{\max }, \\
\mathrm{s}^{-1}\end{array}$ \\
\hline 1,0 & - & 10 & 12 & 2,25 & 7,6 \\
\hline 1,0 & - & 10 & 13 & 3,25 & 7,6 \\
\hline 0,5 & - & 10 & 14 & 2,25 & 40,0 \\
\hline 0,5 & - & 10 & 16 & 3,25 & 40,0 \\
\hline 2,0 & - & 10 & 11 & 2,25 & 4,5 \\
\hline 2,0 & - & 10 & 11.5 & 3,25 & 4,5 \\
\hline 0,25 & - & 8 & 16 & 2,25 & 40,6 \\
\hline 0,25 & - & 8 & 20 & 3,25 & 40,6 \\
\hline 0,25 & $0,25 \mathrm{~d}$ & 10 & 22 & $3,25+0,25 \mathrm{~d}$ & 43,2 \\
\hline 0,25 & $0,5 \mathrm{~d}$ & 10 & 22 & $3,25+0,5 \mathrm{~d}$ & 35,6 \\
\hline 0,5 & $0,5 \mathrm{~d}$ & 10 & 16 & $3,25+0,5 \mathrm{~d}$ & 9,75 \\
\hline 1,0 & $0,5 \mathrm{~d}$ & 10 & 13 & $3,25+0,5 \mathrm{~d}$ & 7,5 \\
\hline
\end{tabular}

the sieve and the average weight of the load is stabilized.

The programmed change in the mass of the load $m_{L}$ is realized by changing the loading rate $V=V_{0}+\Delta V(t)$, where $V_{0}$ is a certain constant component of the loading rate and $\Delta V(t)$ is the deviation of this rate. The maximum deviation of the loading rate varies in different experiments in the range from $0.25 \mathrm{~kg} / \mathrm{s}$ to $0.5 \mathrm{~kg} / \mathrm{s}$. Numerically $\Delta V(t)$ is modeled as a sinusoidal speed increment. The initial mass of the load is $m_{L}=0.25 \mathrm{~kg}$. The parameters of the algorithm for passing through the resonance $\gamma=1.7, T_{\psi}=0.6 \mathrm{~s}$. The value of the steadystate average speed of the rotors $\omega_{r}=70 \mathrm{~s}^{-1}$.

The simulation results are presented in table 2 . The loading rate varies from $0.25 \mathrm{~kg} / \mathrm{s}$ to $2 \mathrm{~kg} / \mathrm{s}$, the time interval in which the mass of the load increases also varies. Table 2 shows: $m_{L m}$ is the average mass of the load af-
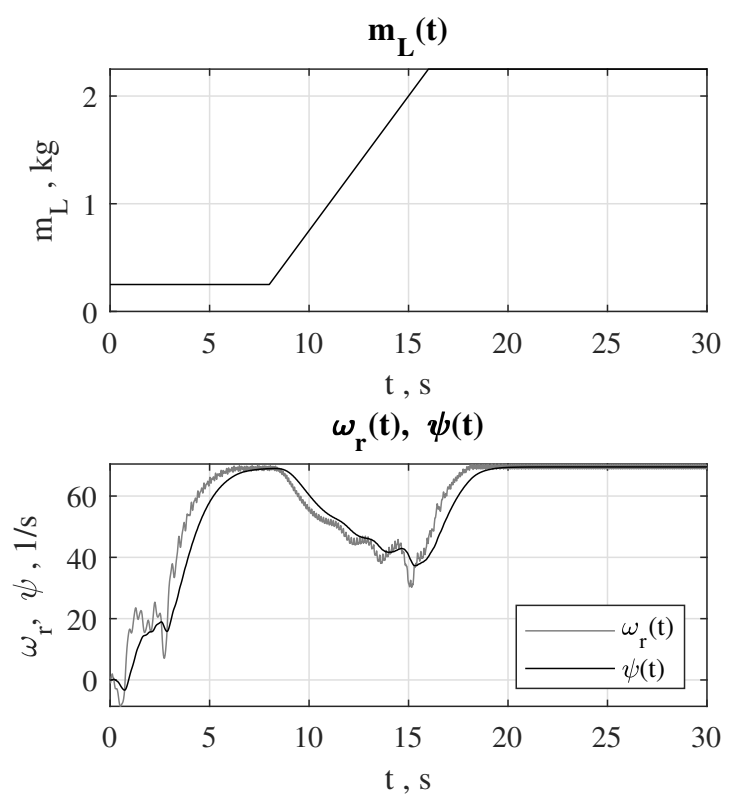

Figure 11. Simulation results for the model with variable load (M3) using algorithm (8) for $V_{0}=0,25 \mathrm{~kg} / \mathrm{s} ; t_{1}=8 \mathrm{~s} ; t_{2}=16 \mathrm{~s}$; $m_{L m}=2,25 \mathrm{~kg}$.
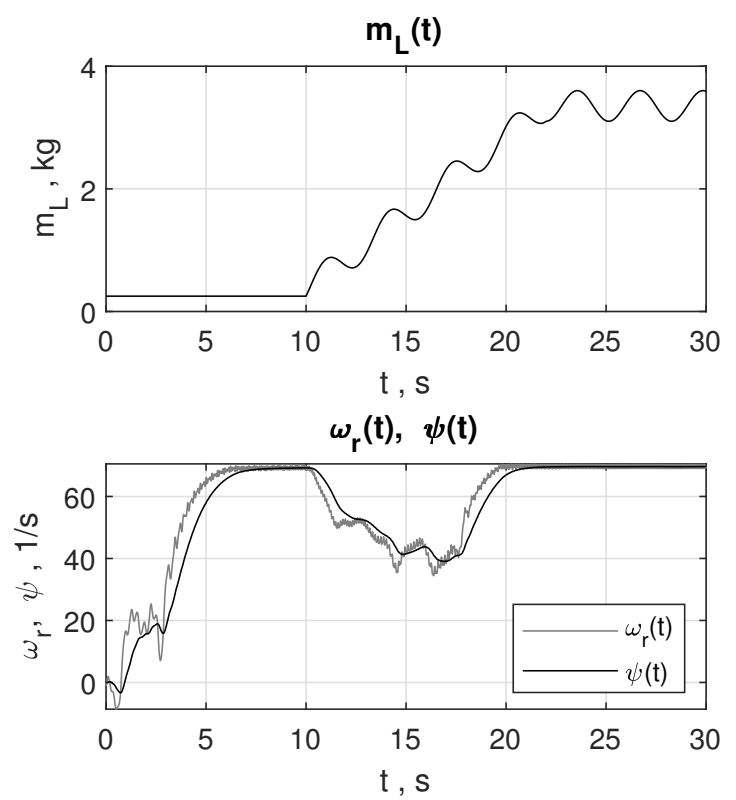

Figure 12. Simulation results for the model with variable load (M3) using algorithm (8) for $V_{0}=0,25 \mathrm{~kg} / \mathrm{s} ; \Delta V=0.5 \sin (2 t) \mathrm{kg} / \mathrm{s}$, $t_{1}=10 \mathrm{~s} ; t_{2}=22 \mathrm{~s} ; m_{L m}=3,25+0,5 \sin 2 t \mathrm{~kg}$. 
ter the instant of time $t_{2}$ and $\Delta \omega_{\max }$ is the value of the maximum drop in the steady-state speed of the rotors after the start of loading. For brevity, the table uses the notation $d=\sin 2 t$.

In fig. 10 the graphs of changes in the following variables are shown:

a) changing the position of the platform horizontally: $x_{c}(t), \mathrm{m}$.

b) change in the vertical position of the platform: $y_{c}(t)$, $\mathrm{m}$;

c) weight of load: $m_{L}, \mathrm{~kg}$;

d) rotor speed: $\omega_{r}=\dot{\varphi}_{r}, \mathrm{~s}^{-1}$ and filter variable $\psi$;

e) graph of the rotor armature current $I_{a}, \mathrm{~A}$;

f) EMF of the thyristor converter $E_{T C}$, V.

In fig. 11 and fig. 12 the graphs of the change in the weight of the load and the rotor speed with varying the parameters of the loading process are shown.

Thus, as the simulation showed, in the time interval starting at the time instant of the beginning of loading and ending when the variable mass of the load enters a stable operating mode, a drop in the rotor speed is observed, which disappears when the average mass of the load stabilizes. Moreover, the magnitude of this decrease does not depend on the duration of the loading and on the steady-state value of the average mass of bulk material loaded on the pallet, but depends only on the average loading speed: the less it is, the greater the drop in the rotor speed. This feature of the control algorithm (8) can be explained by the fact that at the beginning of loading, the total mass of the vibration plant with the load changes, which causes a change in the resonance frequency. However, after some time, the control algorithm works out this disturbance and stabilizes the rotor speed at the same level determined by the $H^{*}$ parameter.

\section{Conclusion}

An algorithm for the passage of the rotor speed through the resonant frequency for a one-rotor vibration unit taking into account the non-stationary attached load has been analyzed by computer simulation. It is shown that the proposed control algorithm can reduce the magnitude of the control action required to pass through the resonance zone. In addition, the algorithm is rather simple and is based on setting just three real parameter values, despite the complexity of the system.

The dependence of the value of the steady-state speeds of the rotors on the level of the desired unit energy has been evaluated, which makes it possible to determine the value of the specified parameter in the control algorithm for practical implementation, depending on the technological task.

It is shown by computer simulation that the steadystate rotor velocities do not depend on the parameters $\gamma$ and $T_{\psi}$, but are determined only by the value of the given total energy $H^{*}$. However, the parameters $\gamma$ and $T_{\psi}$ significantly affect the amplitude of the steady-state armature current, the steady-state EMF of the converter and the synchronization time of the rotors.
As shown by computer simulation, during the time interval starting at the time instant of the beginning of loading and ending when the variable mass of the load enters a stable operating mode, there is a drop in the speeds of the rotors, which disappears when the average mass of the load stabilizes. Moreover, the magnitude of this subsidence depends mainly not on the value of the average mass of bulk material loaded on the pallet, but on the transition time of the installation from the unloaded mode of operation to the mode of operation with the load stabilized by weight.

Discovering the mechanism of such a drop could be a topic of further research. It would be interesting also to extend the obtained results to two-rotor units [Tomchina, 2020] and to the study of vibration fields [Tomchina, 2019].

\section{Acknowledgements}

The work was supported by the Ministry of Science and Higher Education of Russian Federation (075-152021-573).

\section{References}

Andrievsky, B. R., Blekhman, I. I., Bortsov, Y. A., Gavrilov, S. V., Konoplev, V. A., Lavrov, B. P., Polyakhov, N. D., Tomchina, O. P., Fradkov, A. L., and Shestakov, V. M. (2001). Control of mechatronic vibration units (in Russian). Nauka, Saint Petersburg.

Blekhman, I. I. (2000). Vibrational mechanics: nonlinear dynamic effects, general approach, applications. World Scientific.

Blekhman, I. I. (2012). Oscillatory strobodynamics a new area in nonlinear oscillations theory, nonlinear dynamics and cybernetical physics. Cybernetics And Physics, 1(1), pp. 5-10.

Blekhman, I. I., Bortsov, Y. A., Burmistrov, A. A., Fradkov, A. L., Gavrilov, S. V., Kononov, O. A., Lavrov, B. P., Sokolov, P. V., Shestakov, V. M., and Tomchina, O. P. (1999). Computer-controlled vibrational set-up for education and research. In Proc. of 14th IFAC World Congress, vol. M, IFAC, pp. 193-197.

Fradkov, A., Tomchina, O., Tomchin, D., and Gorlatov, D. (2016). Time-varying observer of the supporting body velocity for vibration units. IFAC-PapersOnLine, 49 (14), pp. 18-23.

Fradkov, A. L. (1980). Speed-gradient scheme and its application in adaptive control problems. Automation and Remote Control, 40 (9), pp. 1333-1342.

Fradkov, A. L. (1999). Exploring nonlinearity by feedback. Physica D, 128, pp. 159-168.

Fradkov, A. L., Tomchin, D. A., and Tomchina, O. P. (2014). Controlled passage through resonance for tworotor vibration unit. In Belyaev, A. K., Irschik, H., and Krommer, M., editors, Mechanics and model-based control of advanced engineering systems, pp. 95-102. Springer-Verlag, Wien. 
Fradkov, A. L., Tomchina, O. P., Andrievsky, B., and Boikov, V. I. (2021). Control of phase shift in tworotor vibration units. IEEE Transactions on Control Systems Technology, 29(3), pp. 1316-1322.

Gorlatov, D. V., Tomchin, D. A., and Tomchina, O. P. (2015). Controlled passage through resonance for two-rotor vibration unit: Influence of drive dynamics. IFAC-PapersOnLine, 48 (11), pp. 313-318.

Tomchin, D. A., Tomchina, O. P., and Fradkov, A. L. (2015). Controlled passage through resonance for flexible vibration units. Mathematical Problems in Engineering, 2015.
Tomchina, O. P. (1997). Passing through resonances in vibratory actuators by speed-gradient control and averaging. In Proc. Int. Conf. "Control of Oscillations and Chaos", vol. 1, Saint Petersburg, Russia, IEEE, pp. 138-141.

Tomchina, O. P. (2019). Control of vibrational field in a vibration unit: influence of drive dynamics. Cybernetics and Physics, 8 (4), pp. 298-306.

Tomchina, O. P. (2020). Control of oscillations in tworotor cyberphysical vibration units with time-varying observer. Cybernetics and Physics, 9 (4), pp. 206-213. 